Louisiana State University

LSU Digital Commons

Faculty Publications

Department of Biological Sciences

$11-5-2004$

\title{
STAT 5 activators can replace the requirement of FBS in the adipogenesis of 3T3-L1 cells
}

\author{
William C. Stewart \\ Middle Tennessee State University \\ James E. Baugh \\ Louisiana State University \\ Z. Elizabeth Floyd \\ Louisiana State University \\ Jacqueline M. Stephens \\ Louisiana State University
}

Follow this and additional works at: https://digitalcommons.Isu.edu/biosci_pubs

\section{Recommended Citation}

Stewart, W., Baugh, J., Floyd, Z., \& Stephens, J. (2004). STAT 5 activators can replace the requirement of FBS in the adipogenesis of 3T3-L1 cells. Biochemical and Biophysical Research Communications, 324 (1), 355-359. https://doi.org/10.1016/j.bbrc.2004.09.053

This Article is brought to you for free and open access by the Department of Biological Sciences at LSU Digital Commons. It has been accepted for inclusion in Faculty Publications by an authorized administrator of LSU Digital Commons. For more information, please contact ir@lsu.edu. 


\title{
STAT 5 activators can replace the requirement of FBS in the adipogenesis of $3 \mathrm{~T} 3-\mathrm{L} 1$ cells $^{\text {is }}$
}

\author{
William C. Stewart ${ }^{\mathrm{a}}$, James E. Baugh Jr. ${ }^{\mathrm{b}}$, Z. Elizabeth Floyd ${ }^{\mathrm{b}}$, Jacqueline M. Stephens ${ }^{\mathrm{b}, *}$ \\ a Department of Biology, Middle Tennessee State University, Murfreesboro, TN 37132, United States \\ b Department of Biological Sciences, Louisiana State University, Baton Rouge, LA 70803, United States
}

Received 27 August 2004

Available online 25 September 2004

\begin{abstract}
The 3T3-L1 cells differentiate into fat cells that have many properties of native adipocytes including: substantial lipid accumulation, insulin sensitivity, and the ability to secrete endocrine hormones. A substantial expense in using these cells is fetal bovine serum (FBS), a critical component of efficient adipogenesis. Our recent studies on STAT 5 proteins have revealed that these transcription factors are phosphorylated and translocate to the nucleus immediately after the initiation of differentiation. Studies by several other laboratories also suggest that STAT 5 proteins can have pro-adipogenic properties. Growth hormone (GH) and prolactin (PRL) are both potent activators of STAT 5A and STAT 5B proteins. Since, FBS has high concentrations of GH; we examined the ability of GH to replace FBS as a component of the differentiation cocktail for 3T3-L1 cells. Our studies revealed that FBS was not required for the adipogenesis of 3T3-L1 cells if GH or PRL was added to the differentiation cocktail. Adipogenesis was judged by Oil Red $\mathrm{O}$ staining and expression of adipocyte marker genes. Hence, we have developed a substantially less expensive method for differentiating 3T3-L1 cells without FBS, thiazolidinediones, or expensive cytokines.
\end{abstract}

(C) 2004 Elsevier Inc. All rights reserved.

Keywords: STAT 5; Adipocyte; Adipogenesis

The 3T3-L1 cell line differentiates under the controlled conditions of cell culture from preadipocytes to adipocytes that have the morphological and biochemical properties of fat cells [1,2]. More recent studies have demonstrated that 3-isobutyl-1-methylxanthine (MIX), dexamethasone (DEX), and fetal bovine serum (FBS) are all required components of the differentiation cocktail for the complete adipogenesis of 3T3-L1 cells [3] and for the adipogenesis of non-precursor cells ectopically expressing PPAR $\gamma$ or C/EBPs [4-7].

It is well recognized that several transcription factors are induced during adipocyte differentiation and play a critical role in the regulation of adipocyte gene expres-

\footnotetext{
This work was supported by Grant R01DK52968-05 from the National Institutes of Health to J.M.S.

* Corresponding author. Fax: +1 2255782597.

E-mail address: jsteph1@1su.edu (J.M. Stephens).
}

sion including PPAR $\gamma$, members of the C/EBP family, and members of the forkhead transcription factor family $[8,9]$. It has also been shown that some signal transducers and activators of transcription (STATs), a family of latent transcription factors, can affect adipocyte gene expression. Our previous studies have demonstrated that the expression of STAT5A and STAT5B is induced during differentiation of 3T3-L1 adipocytes [10]. STAT 5 proteins are also induced in the differentiation of human preadipocytes [11]. We have also shown that the induction of both STAT5 proteins correlates with lipid accumulation and the expression of PPAR $\gamma$ [12]. These studies are supported by in vivo experiments that demonstrate that both STAT5A and STAT5B knock-out mice have smaller fat pads than wild-type mice and mice lacking both STAT 5 genes have fat pads one-fifth the size of wild-type mice [13]. In addition, constitutively active STAT 5 can replace the requirement for growth 
hormone in the adipogenesis of 3T3-F442A preadipocytes [14]. More recent studies have shown that ectopic expression of STAT 5A can promote adipogenesis in 3T3-L1 cells [15] and in two different non-precursor cell lines [16]. Both of these studies also demonstrated that STAT 5 proteins are activated, or tyrosine phosphorylated, early after the induction of differentiation and prior to the increase in expression of STAT 5 proteins that occurs with adipogenesis.

Growth hormone $(\mathrm{GH})$ and prolactin (PRL) are both potent activators of STAT 5A and STAT 5B proteins. Since, FBS is known to have high concentrations of GH and stimulation of mature 3T3-L1 adipocytes with FBS can result in the nuclear translocation of STAT 5 proteins [17]; we examined the ability of GH and PRL to replace FBS as a component of the differentiation cocktail for 3T3-L1 cells. Our studies revealed that FBS was not required for the adipogenesis of 3T3-L1 cells if GH or PRL was present in the differentiation cocktail. Adipogenesis was judged by Oil Red O staining and expression of adipocyte marker genes. In summary, we have developed a less expensive method for differentiating 3T3-L1 cells and have clearly demonstrated that STAT 5 activators can promote the adipogenesis of 3T3L1 cells.

\section{Materials and methods}

Materials. Dulbecco's modified Eagle's media (DMEM) were purchased from Life Technologies. Bovine and fetal bovine (FBS) sera were purchased from Sigma and Invitrogen, respectively. Porcine growth hormone and sheep prolactin were purchased from Sigma. FAS monoclonal antibody was purchased from BD Transduction laboratories. Both the PPAR $\gamma$ monoclonal antibody and the STAT 5A polyclonal antibody were purchased from Santa Cruz. HRP-conjugated secondary antibodies were purchased from Jackson Immunoresearch. Enhanced chemiluminescence (ECL) kit was purchased from Pierce. Nitrocellulose and Zeta Probe-GT membranes were purchased from Bio-Rad.

Cell culture. Murine 3T3-L1 preadipocytes were plated and grown to 2 days post-confluence in DMEM with $10 \%$ bovine serum. Medium was changed every $48 \mathrm{~h}$. Cells were induced to differentiate by changing the medium to DMEM containing $10 \%$ fetal bovine serum, $0.5 \mathrm{mM}$ of 3-isobutyl-1-methylxanthine, $1 \mu \mathrm{M}$ dexamethasone, and $1.7 \mu \mathrm{M}$ insulin. After $48 \mathrm{~h}$ this medium was replaced with DMEM supplemented with $10 \% \mathrm{FBS}$, and cells were maintained in this medium until utilized for experimentation. Cells that were induced to differentiate in the absence of FBS contained either $0.3 \%$ BSA or $5 \%$ bovine serum and were never exposed to FBS.

Preparation of whole cell extracts. Monolayers of 3T3-L1 preadipocytes or adipocytes were rinsed with phosphate-buffered saline (PBS) and then harvested in a non-denaturing buffer containing $150 \mathrm{mM} \mathrm{NaCl}, 10 \mathrm{mM}$ Tris, $\mathrm{pH}$ 7.4, $1 \mathrm{mM}$ EGTA, $1 \mathrm{mM}$ EDTA, $1 \%$ Triton X-100, 0.5\% Igepal CA-630 (Nonidet P-40), $1 \mu \mathrm{M}$ PMSF, $1 \mu \mathrm{M}$ pepstatin, 50 trypsin inhibitory milliunits of aprotinin, and $10 \mu \mathrm{M}$ leupeptin, and $2 \mathrm{mM}$ sodium vanadate. Samples were extracted for $30 \mathrm{~min}$ on ice and centrifuged at $15,000 \mathrm{rpm}$ at $4{ }^{\circ} \mathrm{C}$ for $15 \mathrm{~min}$. Supernatants containing whole cell extracts were analyzed for protein content using a BCA kit (Pierce) according to the manufacturer's instructions.
Gel electrophoresis and Western blot analysis. Proteins were separated in $5 \%, 7.5 \%, 10 \%$ or $12 \%$ polyacrylamide (acrylamide from National Diagnostics) gels containing sodium dodecyl sulfate (SDS) according to Laemmli [18] and transferred to nitrocellulose membrane in $25 \mathrm{mM}$ Tris, $192 \mathrm{mM}$ glycine, and $20 \%$ methanol. Following transfer, the membrane was blocked in $4 \%$ fat-free milk for $1 \mathrm{~h}$ at room temperature. Results were visualized with HRP-conjugated secondary antibodies and enhanced chemiluminescence.

Oil Red $O$ staining. Cell monolayers were rinsed twice with phosphate-buffered saline and fixed overnight in Baker's formalin at $4{ }^{\circ} \mathrm{C}$. After fixing, the Baker's was aspirated and a filtered 60\% Oil Red O solution in distilled water, made from a stock of $0.70 \mathrm{~g}$ Oil Red $\mathrm{O}$ in $200 \mathrm{ml}$ isopropanol, was added to the plates. The plates were placed on an orbital shaker for $1 \mathrm{~h}$ and then rinsed with $70 \%$ ethanol to remove background Oil Red O stain.

$R N A$ analysis. Total RNA was isolated from cell monolayers with Trizol according to manufacturer's instructions with minor modifications. For Northern blot analysis, $20 \mu \mathrm{g}$ of total RNA was denatured in formamide and electrophoresed through a formaldehyde/agarose gel. The RNA was transferred to Zeta Probe-GT, cross-linked, hybridized, and washed as previously described [19]. aP2/422 cDNA was labeled by random priming using the Klenow fragment and $\left[\alpha-{ }^{32} \mathrm{P}\right] \mathrm{dATP}$.

\section{Results}

We have recently shown that STAT 5 proteins are phosphorylated rapidly after the induction of adipogenesis of 3T3-L1 cells when the standard induction cocktail is used [16]. STAT 5 proteins remain phosphorylated for $3 \mathrm{~h}$ after the induction of adipogenesis which precedes the induction in expression of numerous adipocyte transcription factors, including PPAR $\gamma$. As shown in Fig. 1, the induction of PPAR $\gamma$ occurs after STAT 5 activation and prior to the increase in STAT 5 expression which occurs during adipogenesis of 3T3-L1 cells [10].

To study STAT 5 activation during adipocyte differentiation, we induced 3T3-L1 cells to differentiate in the absence of FBS. We supplemented the standard induction cocktail with cardiotrophin-1 (CT-1) a potent activator of STATs 1 and 3 that does not affect the adipogenesis of 3T3-L1 cells [20]. We also supplemented the cocktail lacking FBS with GH. As shown by Oil Red O staining in Fig. 2, cells lacking FBS did not accumulate lipid. Exposure to CT-1 did not result in any changes in lipid accumulation. However, cells lacking FBS that were treated with $\mathrm{GH}$ had a marked increase in lipid accumulation as was observed with cells that were exposed to the normal differentiation cocktail (+FBS). These cells were stained $96 \mathrm{~h}$ after the induction of differentiation. CT-1 and GH were present only during the first $48 \mathrm{~h}$ when cells were exposed to the induction cocktail.

To confirm that STAT 5 activators could promote adipogenesis, we also examined the expression of ap2/ 422 mRNA and fatty acid synthase (FAS) protein expression at two different times after the induction of 


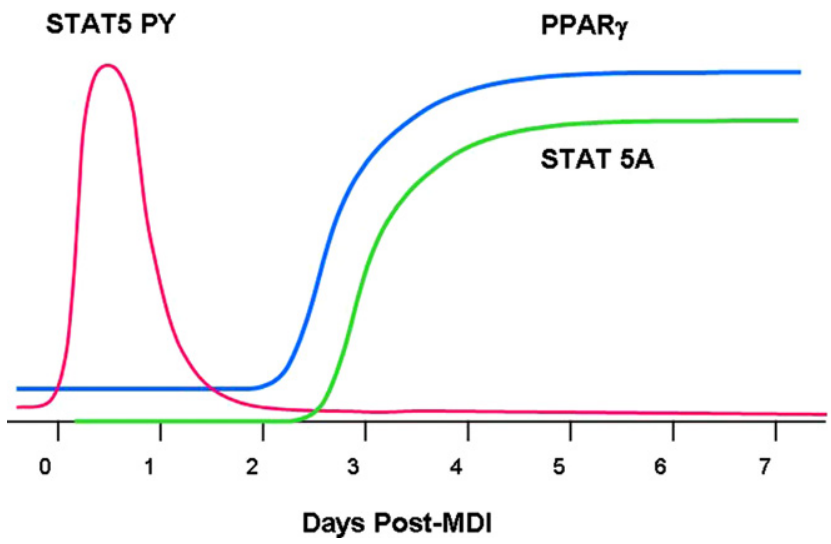

Fig. 1. The activation, or tyrosine phosphorylation of STAT 5 proteins, precedes the induction in expression of PPAR $\gamma$ and STAT 5 proteins during the adipogenesis of 3T3-L1 cells.

-FBS

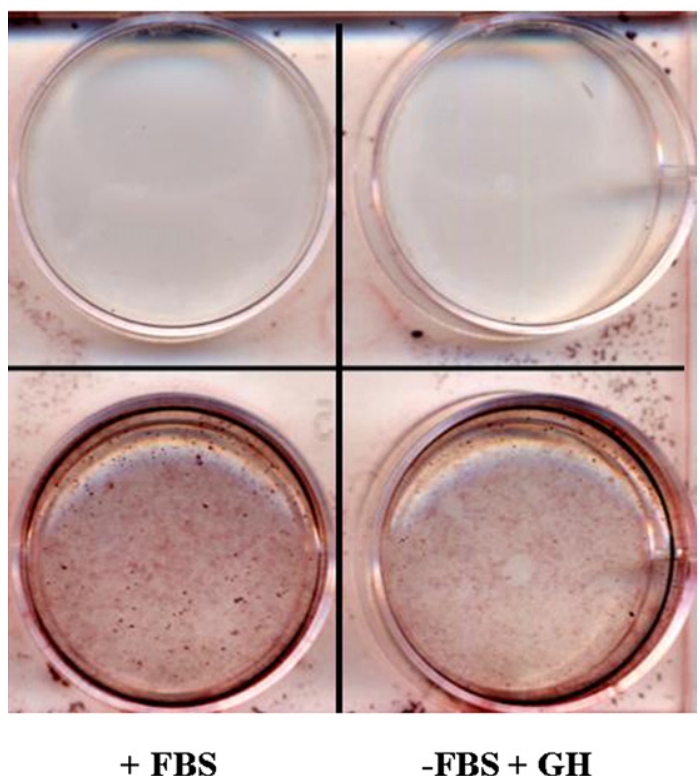

Fig. 2. STAT 5 activators, not a STAT 1 and 3 activator, promote lipid accumulation of 3T3-L1 cells in the absence of FBS. Cell monolayers were subjected to Oil Red $\mathrm{O}$ staining $96 \mathrm{~h}$ after exposure to the standard differentiation cocktail (+FBS) or with a cocktail lacking FBS (-FBS). Some cells lacking FBS were supplemented with $50 \mathrm{nM}$ growth hormone $(\mathrm{GH})$ or $0.1 \mathrm{nM}$ cardiotrophin $(\mathrm{CT}-1)$ for the $48 \mathrm{~h}$ when cells were exposed to MIX, DEX, and INS.

differentiation with cocktails lacking FBS that were supplemented with GH or PRL. As shown in Fig. 3, ap2/ $422 \mathrm{mRNA}$ is detectable $48 \mathrm{~h}$ after the induction of differentiation with the normal cocktail (CTL) or cocktails lacking FBS that were supplemented with either GH or PRL. A similar pattern was also observed at $96 \mathrm{~h}$. Interestingly, the expression of FAS protein was not different for the four different cocktails at $48 \mathrm{~h}$. However, at $96 \mathrm{~h}$ after the initiation of differentiation, there was abundant

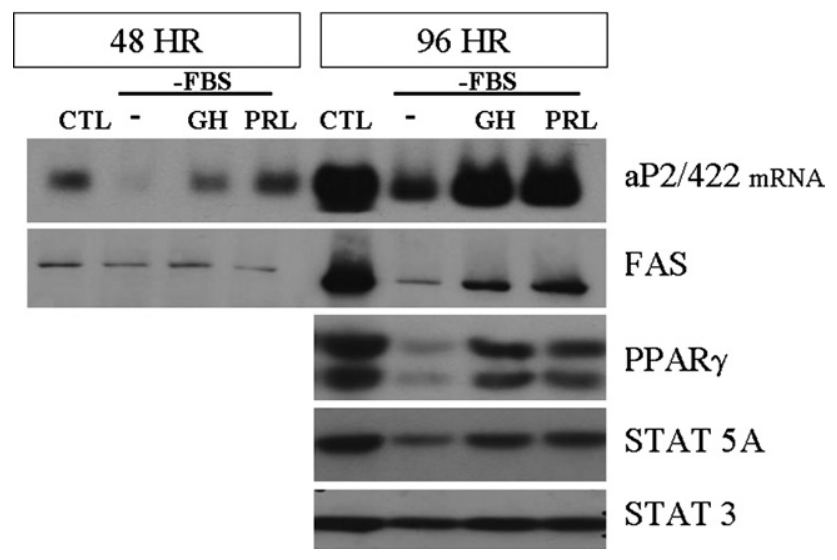

Fig. 3. STAT 5 activators induce adipogenesis of 3T3-L1 cells in the absence of FBS. Total RNA and whole cell extracts were prepared from 3T3-L1 cells that were induced to differentiate with the normal induction cocktail in the presence or absence of fetal bovine serum (FBS). Cells lacking FBS contained $0.3 \%$ fatty acid-free and growth factor depleted BSA. Some cells were also exposed to $50 \mathrm{nM} \mathrm{GH}$ or $22 \mathrm{nM}$ PRL at the initiation of differentiation and $24 \mathrm{~h}$ after the initiation of differentiation. One hundred micrograms of each extract was separated by SDS-PAGE, transferred to nitrocellulose, and subjected to Western blot analysis. The detection system was horseradish peroxidase-conjugated secondary antibodies (Sigma) and enhanced chemiluminescence (Pierce). Twenty micrograms of total RNA was electrophoresed, transferred to Nylon, and subjected to Northern blot analysis to examine aP2/422 expression.

expression of these adipocyte markers induced by the normal cocktail. In addition, we observed a considerable level of FAS protein in cells that were supplemented with GH or PRL. However, the levels were less than that observed for the normal cocktail. We also examined the expression of PPAR $\gamma$ and STAT5A, two transcription factors known to be induced during adipocyte differentiation. As shown in Fig. 3, the expression of these transcription factors was induced during adipogenesis and the pattern of expression was very similar to what we observed for both ap2/422 and FAS. However, the levels of STAT 3 were not modulated under these conditions.

These experiments suggested that an additional component of the serum can affect the differentiation of 3T3L1 cells. Hence, we performed a similar experiment, but used 5\% calf serum (CS) in cells that were not treated with the normal induction cocktail of $10 \%$ FBS. One week after the induction of differentiation, two plates from each condition were harvested to examine the expression of PPAR $\gamma$ and STAT5A. As shown in Fig. 4, cells lacking FBS, but containing 5\% CS did not differentiate as judged by expression of PPAR $\gamma$ and STAT5A. However, cells supplemented with either GH or PRL in the presence of 5\% calf serum were able to induce expression of these transcription factors at levels observed with normal differentiation cocktail. Also, the levels of STAT 3 expression were not regulated under these conditions. 


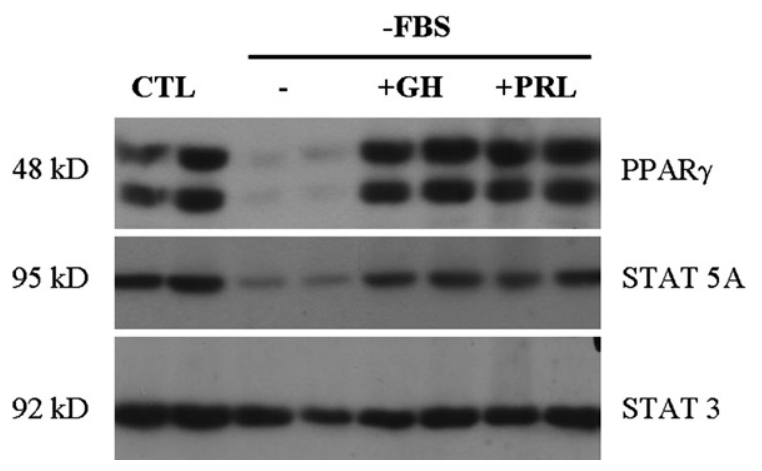

Fig. 4. GH or PRL can mimic the effects of FBS on the adipogenesis of 3T3-L1 cells. Whole cell extracts were prepared from 3T3-L1 cells 1 week after the cells were induced to differentiate with the normal induction cocktail in the presence or absence of fetal bovine serum. Cells that lacked FBS were supplemented with $5 \%$ calf serum and some cells were also exposed to $50 \mathrm{nM} \mathrm{GH}$ or $22 \mathrm{nM}$ PRL at the initiation of differentiation and $24 \mathrm{~h}$ after the initiation of differentiation. One hundred micrograms of each extract was separated by SDS-PAGE, transferred to nitrocellulose, and subjected to Western blot analysis. Samples were processed and results were visualized as described in Fig. 3.

\section{Discussion}

Studies by many laboratories have shown that the expression of STAT 5 proteins correlates with adipogenesis. Our recent studies have also demonstrated that STAT 5 proteins are tyrosine phosphorylated immediately after the initiation of differentiation. Hence, in this study we examined the ability of STAT 5 activators to replace FBS as a necessary component of the induction cocktail needed to induce the adipogenesis of 3T3-L1 cells. We hypothesized that the role of the FBS in inducing differentiation in vitro may be to activate STAT 5 proteins. Our studies revealed that two STAT 5 activators, GH and PRL, could result in lipid accumulation and the expression of adipocyte markers in the absence of FBS. However, the levels of lipid accumulation and fat cell gene expression were lower than observed in cells differentiated with $10 \%$ FBS. Yet, these STAT 5 activators could confer adipogenesis of 3T3-L1 in the presence of the $5 \%$ calf serum which is significantly less expensive than fetal calf serum. In this experiment, either GH or PRL induced PPAR $\gamma$ to levels observed with the normal induction cocktail. Our results indicate STAT 5 activators can replace the presence of FBS for in vitro differentiation of 3T3-L1 cells (Figs. 2 and 3), but some other factor present in calf serum is needed for these STAT 5 activators to induce maximal expression of adipocyte markers (Fig. 4). These effects appear to be specific for STAT 5 activators, since CT-1, a potent STAT 1 and STAT 3 activator cannot confer adipogenesis in the absence of FBS (Fig. 3). Future studies will focus on what other factor(s) or calf serum components in addition to STAT 5 activators are required to induce fat cell differentiation of 3T3-L1 cells. In conclusion, we have developed a substantially less expensive method for differentiating 3T3-L1 cells without FBS, thiazolidinediones, or expensive cytokines and have clearly demonstrated that STAT 5 activators promote adipogenesis.

\section{Acknowledgments}

We are grateful to Pat Arbour-Reily for her technical assistance and to Jill Robertson from MTSU for her participation in this project. We are also thankful to Mike Gibbs at Pfizer for the idea to pursue this line of investigation.

\section{References}

[1] H. Green, O. Kehinde, An established preadipose cell line and its differentiation in culture. II. Factors affecting the adipose conversion, Cell 5 (1975) 19-27.

[2] H. Green, M. Meuth, An established pre-adipose cell line and its differentiation in culture, Cell 3 (1974) 127-133.

[3] W.C. Yeh, Z. Cao, M. Classon, S.L. McKnight, Cascade regulation of terminal adipocyte differentiation by three members of the C/EBP family of leucine zipper proteins, Genes Dev. 9 (1995) $168-181$.

[4] F.T. Lin, M.D. Lane, CCAAT/enhancer binding protein alpha is sufficient to initiate the 3T3-L1 adipocyte differentiation program, Proc. Natl. Acad. Sci. USA 91 (1994) 8757-8761.

[5] P. Tontonoz, E. Hu, B.M. Spiegelman, Stimulation of adipogenesis in fibroblasts by PPAR gamma 2, a lipid-activated transcription factor [published erratum appears in Cell (1995) Mar 24;80(6):following 957], Cell 79 (1994) 1147-1156.

[6] Z. Wu, N.L. Bucher, S.R. Farmer, Induction of peroxisome proliferator-activated receptor gamma during the conversion of 3T3 fibroblasts into adipocytes is mediated by C/EBPbeta, C/ EBPdelta, and glucocorticoids, Mol. Cell. Biol. 16 (1996) 41284136.

[7] Z. Wu, Y. Xie, N.L. Bucher, S.R. Farmer, Conditional ectopic expression of C/EBP beta in NIH-3T3 cells induces PPAR gamma and stimulates adipogenesis, Genes Dev. 9 (1995) 2350 2363.

[8] E.D. Rosen, Molecular mechanisms of adipocyte differentiation, Ann. Endocrinol. (Paris) 63 (2002) 79-82.

[9] H.S. Camp, D. Ren, T. Leff, Adipogenesis and fat-cell function in obesity and diabetes, Trends. Mol. Med. 8 (2002) 442-447.

[10] J.M. Stephens, R.F. Morrison, P.F. Pilch, The expression and regulation of STATs during 3T3-L1 adipocyte differentiation, J. Biol. Chem. 271 (1996) 10441-10444.

[11] J.B. Harp, D. Franklin, A.A. Vanderpuije, J.M. Gimble, Differential expression of signal transducers and activators of transcription during human adipogenesis, Biochem. Biophys. Res. Commun. 281 (2001) 907-912.

[12] W.C. Stewart, R.F. Morrison, S.L. Young, J.M. Stephens, Regulation of signal transducers and activators of transcription (STATs) by effectors of adipogenesis: coordinate regulation of STATs 1, 5A, and 5B with peroxisome proliferator-activated receptor-gamma and C/AAAT enhancer binding protein-alpha, Biochim. Biophys. Acta 1452 (1999) 188-196.

[13] S. Teglund, C. McKay, E. Schuetz, J.M. van Deursen, D. Stravopodis, D. Wang, M. Brown, S. Bodner, G. Grosveld, J.N. 
Ihle, Stat5a and Stat5b proteins have essential and nonessential, or redundant, roles in cytokine responses, Cell 93 (1998) 841-850.

[14] C.A. Shang, M.J. Waters, Constitutively active signal transducer and activator of transcription 5 can replace the requirement for growth hormone in adipogenesis of 3T3-F442A preadipocytes, Mol. Endocrinol. 17 (2003) 2494-2508.

[15] R. Nanbu-Wakao, Y. Morikawa, I. Matsumura, Y. Masuho, M.A. Muramatsu, E. Senba, H. Wakao, Stimulation of 3T3-L1 adipogenesis by signal transducer and activator of transcription 5 , Mol. Endocrinol. 16 (2002) 1565-1576.

[16] Z.E. Floyd, J.M. Stephens, STAT5A promotes adipogenesis in nonprecursor cells and associates with the glucocorticoid receptor during adipocyte differentiation, Diabetes 52 (2003) 308314.
[17] J.P. Balhoff, J.M. Stephens, Highly specific and quantitative activation of STATs in adipocytes, Biochem. Biophys. Res. Commun. 247 (1998) 894-900.

[18] U.K. Laemmli, Cleavage of structural proteins during the assembly of the head of bacteriophage T4, Nature 227 (1970) 680-685.

[19] J.M. Stephens, P.H. Pekala, Transcriptional repression of the C/ EBP-alpha and GLUT4 genes in 3T3-L1 adipocytes by tumor necrosis factor-alpha. Regulations is coordinate and independent of protein synthesis, J. Biol. Chem. 267 (1992) 13580-13584.

[20] S. Zvonic, P. Cornelius, W.C. Stewart, R.L. Mynatt, J.M. Stephens, The regulation and activation of ciliary neurotrophic factor signaling proteins in adipocytes, J. Biol. Chem. 278 (2003) 2228-2235. 